\title{
CHEMICAL POTENTIAL DERIVATIVE AS HALLMARK FOR PHASE TRANSITIONS
}

\author{
M. Pietruszka \\ Faculty of Biology and Environmental Protection, University of Silesia \\ Jagiellońska 28, 40-032 Katowice, Poland \\ AND M. MATLAK* \\ Institute of Physics, University of Silesia \\ Uniwersytecka 4, 40-007 Katowice, Poland \\ (Received July 27, 1999; in final form October 15, 1999)
}

We study antiferromagnetic properties of the two-band extended $s-f$ model with fluctuating valence in the context of two mutually bound new effects of chemical potential critical behaviour, as well as of critical electron redistribution. In order to exemplify both phenomena we build phase diagrams of the system displaying the dependence of the critical Néel temperatures $\left(T_{\mathrm{N}}\right)$ of the system versus $4 f(5 f)$ level positions. The phase diagram consists of two different areas corresponding to antiferromagnetic and paramagnetic phases. We plot the magnetizations and the correlation functions of the system as functions of temperature. Next, we investigate the temperature dependence of the relative average occupation numbers $\Delta n^{f(d)}$ and the chemical potential $\Delta \mu$ for a given $4 f(5 f)^{\prime}$ level position $E_{f}$. Plotting this quantities along the $E_{f}$ cross-section lines we observe small (of the order of $10^{-4}-10^{-3}$ ) but well localized kinks exactly at the Néel temperature $T_{\mathrm{N}}$. Last but not least, we plot the first derivative of the chemical potential $\mathrm{d} \mu / \mathrm{d} T$ which, as it shows clearly visible jumps at $T_{\mathrm{N}}$, may turn out to be very accurate and sensitive (auxiliary) tool to find critical temperatures of the considered system. Moreover, we plot the difference $\mu_{\mathrm{AF}}-\mu_{\mathrm{PARA}}$ where we subtract a chemical potential value of a reference paramagnetic sample from the actual value of the antiferromagnetic system. Also in this case we report the observation of discontinuous change in slope at $T_{N}$. Our observations can be extended to point out to a new practical possibility of how to find experimentally the critical temperatures of the antiferromagnetic systems exclusively from the chemical potential measurements. We expect that the same type of measurement, according to our recent and present results, would also apply to all types of critical phenomena in real solids.

PACS numbers: $64.60 .-\mathrm{i}, 75.40 . \mathrm{Cx}, 75.50 . \mathrm{Ee}$

\footnotetext{
*e-mail: matlak@server.phys.us.edu.pl
} 


\section{Introduction}

The magnetic properties of the $4 f$ (lanthanide) systems like $\mathrm{Ce}, \mathrm{Pr}, \mathrm{Sm}, \mathrm{Eu}$, $\mathrm{Tm}$, and $\mathrm{Yb}$-based materials and of the $5 f$ systems (actinide based materials) are extensively reviewed in Refs. [1-6]. One can find, among them, many intermediate valence magnetic materials with interesting and unusual physical properties. The physical properties of the mentioned materials can successfully be explained with the use of the extended $s-f$ model (see e.g. Refs. [7-10]). However, in view of the results of the recent papers, Refs. [11-14], concerning the straightforward relations between chemical potential temperature dependence and phase transitions in the electronic system, it seems to be interesting to see whether for magnetic systems exhibiting fluctuating valence the critical behaviour of the chemical potential can also be able to report on phase transitions in such systems. In the present paper we show that for antiferromagnets exhibiting fluctuating valence, described by the extended $s-f$ model, Refs. [7-10], the phase transition antiferromagnet-paramagnet at Néel's temperature $T_{N}$ can clearly be detected (similarly to other types of phase transitions, Refs. [11-14]) exclusively from the plot of the chemical potential versus temperature, because of visible kinks at $T=T_{\mathrm{N}}$.

\section{Model and results}

In order to investigate the antiferromagnetism in $4 f(5 f)$ systems we consider two-sublattice version of the generalized $s-f$ model (cf. e.g. Ref. [10]). The Hamiltonian for this case can be written in the following way (grand canonical ensemble):

$$
H-\mu N=H_{f A}+H_{f B}+H_{f A B}+H_{f d A}+H_{f d B}+H_{d A B}-\mu N,
$$

where

$$
\begin{aligned}
H_{f \alpha} & =E_{f} \sum_{i_{\alpha} \sigma} n_{i_{\alpha} \sigma}^{f}+U_{f} \sum_{i_{\alpha}} n_{i_{\alpha} \uparrow}^{f} n_{i_{\alpha} \downarrow}^{f} \\
H_{f A B} & =\sum_{i_{A} j_{B} \sigma} t_{i_{A} j_{B}}^{f} f_{i_{A} \sigma}^{+} f_{j_{B} \sigma}+\sum_{i_{B} j_{A} \sigma} t_{i_{B} j_{A}}^{f} f_{i_{B} \sigma}^{+} f_{j_{A} \sigma}, \\
H_{f d \alpha} & =-\frac{g}{2} \sum_{i_{\alpha}}\left(f_{i_{\alpha} \uparrow}^{+} f_{i_{\alpha} \downarrow} d_{i_{\alpha} \downarrow}^{+} d_{i_{\alpha} \uparrow}+f_{i_{\alpha} \downarrow}^{+} f_{i_{\alpha} \uparrow} d_{i_{\alpha} \uparrow}^{+} d_{i_{\alpha} \downarrow}\right) \\
& -\frac{g}{4} \sum_{i_{\alpha}}\left(n_{i_{\alpha} \uparrow}^{f}-n_{i_{\alpha} \downarrow}^{f}\right)\left(n_{i_{\alpha} \uparrow}^{d}-n_{i_{\alpha} \downarrow}^{d}\right) \\
& +V \sum_{i_{\alpha} \sigma}\left(f_{i_{\alpha} \sigma}^{+} d_{i_{\alpha} \sigma}+d_{i_{\alpha} \sigma}^{+} f_{i_{\alpha} \sigma}\right), \quad \alpha=A, B, \\
H_{d A B} & =t_{0} \sum_{i_{A} \sigma} n_{i_{A} \sigma}^{d}+t_{0} \sum_{i_{B} \sigma} n_{i_{B} \sigma}^{d}+\sum_{i_{A} j_{B} \sigma} t_{i_{A} j_{B}}^{d} d_{i_{A} \sigma}^{+} \sigma d_{j_{B} \sigma} \\
& +\sum_{i_{B} j_{A} \sigma} t_{i_{B} j_{A}}^{d} d_{i_{B} \sigma}^{+} d_{j_{A} \sigma}
\end{aligned}
$$


and

$$
N=\sum_{i_{A} \sigma}\left(n_{i_{A} \sigma}^{f}+n_{i_{A} \sigma}^{d}\right)+\sum_{i_{B} \sigma}\left(n_{i_{B} \sigma}^{f}+n_{i_{B} \sigma}^{d}\right)
$$

The two types of lattice sites $i_{\alpha}, j_{\alpha}(\alpha=A, B)$ are taken into account. The $4 f(5 f)$ subsystem is described by the Hamiltonian, Eqs. (2) and (3), where $E_{f}$ denotes the position of the $4 f(5 f)$ band gravity centre, $U_{f}$ stands for the intrasite Coulomb repulsion, $t_{i_{A} j_{B}}^{f}$ and $t_{i_{B} j_{A}}^{f}$ are hopping integrals of the narrow $4 f(5 f)$ band. The first two terms of the Hamiltonian, Eq. (4), describe the local exchange interaction of the localized $4 f(5 f)$ magnetic moments with conduction electrons (the coupling constant $g$ ) and the third term - the hybridization between $4 f(5 f)$ electrons and conduction electrons. The subsystem of conduction electrons is described by the Hamiltonian, Eq. (5); $t_{0}$ denotes the centre of gravity position of the conduction band, $t_{i_{A} j_{B}}^{d}$ and $t_{i_{B} j_{A}}^{d}$ are hopping integrals for these electrons; $\mu$ denotes the chemical potential.

To simplify further calculations we perform the mean field approximation in Eq. (4), similarly to Ref. [10]. We can write

and

$$
f_{i_{\alpha} \sigma}^{+} f_{i_{\alpha}-\sigma} d_{i_{\alpha}-\sigma}^{+} d_{i_{\alpha} \sigma} \approx-X_{\sigma}^{\alpha} d_{i_{\alpha}-\sigma}^{+} f_{i_{\alpha}-\sigma}-\left(X_{-\sigma}^{\alpha}\right)^{*} f_{i_{\alpha} \sigma}^{+} d_{i_{\alpha} \sigma}
$$

where

$$
\left(n_{i_{\alpha} \uparrow}^{f}-n_{i_{\alpha} \downarrow}^{f}\right)\left(n_{i_{\alpha} \uparrow}^{d}-n_{i_{\alpha} \downarrow}^{d}\right) \approx 2 m_{\alpha}^{f}\left(n_{i_{\alpha} \uparrow}^{d}-n_{i_{\alpha} \downarrow}^{d}\right)+2 m_{\alpha}^{d}\left(n_{i_{\alpha} \uparrow}^{f}-n_{i_{\alpha} \downarrow}^{f}\right),
$$

$$
X_{\sigma}^{\alpha}=\left\langle d_{i_{\alpha} \sigma}^{+} f_{i_{\alpha} \sigma}\right\rangle, \quad m_{\alpha}^{f(d)}=\frac{1}{2}\left(\left\langle n_{i_{\alpha} \uparrow}^{f(d)}\right\rangle-\left\langle n_{i_{\alpha} \downarrow}^{f(d)}\right\rangle\right),
$$

and $\alpha=A, B$. For the antiferromagnetic case, we are interested in, we assume that the magnetizations fulfil the relations

$$
m_{A}^{f(d)}=m_{f(d)}, \quad m_{B}^{f(d)}=-m_{f(d)} .
$$

By using Eqs. (7)-(9), and (10) we get simplified form of Hamiltonian (1) in the form

$$
\begin{aligned}
H- & \mu N=\sum_{\alpha=A, B}\left(\sum_{i_{\alpha} \sigma} E_{\sigma}^{f \alpha} n_{i_{\alpha} \sigma}^{f}+U_{f} \sum_{i_{\alpha}} n_{i_{\alpha} \dagger}^{f} n_{i_{\alpha} \downarrow}^{f}\right) \\
& +\sum_{\alpha=A, B} \sum_{i_{\alpha} \sigma}\left[V_{\sigma}^{\alpha} f_{i_{\alpha} \sigma}^{+} d_{i_{\alpha} \sigma}+\left(V_{\sigma}^{\alpha}\right)^{*} d_{i_{\alpha} \sigma}^{+} f_{i_{\alpha} \sigma}\right]+\sum_{i_{A} j_{B} \sigma} t_{i_{A} j_{B}}^{f} f_{i_{A} \sigma}^{+} f_{j_{B} \sigma} \\
& +\sum_{i_{B} j_{A} \sigma} t_{i_{B} j_{A}}^{f} f_{i_{B} \sigma}^{+} f_{j_{A} \sigma}+\sum_{i_{A} j_{B} \sigma} t_{i_{A} j_{B}}^{d} d_{i_{A} \sigma}^{+} d_{j_{B} \sigma}+\sum_{i_{B} j_{A} \sigma} t_{i_{B} j_{A}}^{d} d_{i_{B} \sigma}^{+} d_{j_{A} \sigma} \\
& +\sum_{\alpha=A, B} \sum_{i_{\alpha} \sigma} t_{\sigma}^{\alpha} n_{i_{\alpha} \sigma}^{d}
\end{aligned}
$$

where

$$
\begin{aligned}
& E_{\uparrow}^{f A}=E_{f}-\mu-\frac{g}{2} m_{d}\left(-\frac{J_{0}}{2} m_{f}\right) \equiv E_{\uparrow}^{f}\left(-\frac{J_{0}}{2} m_{f}\right), \\
& E_{\downarrow}^{f A}=E_{f}-\mu+\frac{g}{2} m_{d}\left(+\frac{J_{0}}{2} m_{f}\right) \equiv E_{\downarrow}^{f}\left(+\frac{J_{0}}{2} m_{f}\right),
\end{aligned}
$$




$$
\begin{aligned}
& E_{\uparrow}^{f B}=E_{f}-\mu+\frac{g}{2} m_{d}\left(+\frac{J_{0}}{2} m_{f}\right) \equiv E_{\downarrow}^{f}\left(+\frac{J_{0}}{2} m_{f}\right), \\
& E_{\downarrow}^{f B}=E_{f}-\mu-\frac{g}{2} m_{d}\left(-\frac{J_{0}}{2} m_{f}\right) \equiv E_{\uparrow}^{f}\left(-\frac{J_{0}}{2} m_{f}\right), \\
& V_{\sigma}^{A}=V+\frac{g}{2} X_{-\sigma}^{A}, \quad V_{\sigma}^{B}=V+\frac{g}{2} X_{-\sigma}^{B}, \\
& t_{\uparrow}^{A}=-\mu-\frac{g}{2} m_{f} \equiv t_{\uparrow}, \quad t_{\downarrow}^{A}=-\mu+\frac{g}{2} m_{f} \equiv t_{\downarrow}, \\
& t_{\uparrow}^{B}=-\mu+\frac{g}{2} m_{f} \equiv t_{\downarrow}, \quad t_{\downarrow}^{B}=-\mu-\frac{g}{2} m_{f} \equiv t_{\uparrow}
\end{aligned}
$$

(the terms in braces in Eq. (12) appear when we utilize additionally Ising-like direct exchange term in the Hamiltonian, introducing in this way direct $4 f(5 f)-4 f(5 f)$ exchange interaction).

To find the thermodynamic properties of the system described by the Hamiltonian from Eq. (1) we can use the simplified form, Eq. (11). The calculation method used here is similar to Ref. [10]. The only modifications concern two sublattices instead of one. We write first the exact Dyson equations for the case of $U_{f}=0$ and, next, the presence of the Coulomb repulsion can be taken into account by the renormalization of the free propagator

$$
\frac{1}{E-E_{A, B \sigma}^{f}} \rightarrow \frac{1-\left\langle n_{A, B-\sigma}^{f}\right\rangle}{E-E_{A, B \sigma}^{f}}+\frac{\left\langle n_{A, B-\sigma}^{f}\right\rangle}{E-E_{A, B \sigma}^{f}-U_{f}}
$$

in the corresponding Dyson equations (see Ref. [10]). Using this method we can calculate all Green functions we need. To determine the chemical potential $\mu$ with the help of the following constraint:

$$
\sum_{\sigma}\left(\left\langle n_{\alpha \sigma}^{f}\right\rangle+\left\langle n_{\alpha \sigma}^{d}\right\rangle\right)=n, \quad \alpha=A, B
$$

where $n$ is the average number of electrons per atom (the same for both $A$ and $B$ sublattices) we need the following Green functions: $\left\langle\left\langle f_{\alpha \sigma} \mid f_{\alpha \sigma}^{+}\right\rangle\right\rangle_{k_{, E}},\left\langle\left\langle d_{\alpha \sigma} \mid d_{\alpha \sigma}^{+}\right\rangle\right\rangle_{k, E}$, $\left\langle\left\langle f_{\alpha \sigma} \mid d_{\alpha \sigma}^{+}\right\rangle\right\rangle_{k, E}$ and $\left\langle\left\langle d_{\alpha \sigma} \mid f_{\alpha \sigma}^{+}\right\rangle\right\rangle_{k, E}, \alpha=A, B$. Hereafter we assume that both $V_{\sigma}^{A}$ and $V_{\sigma}^{B}(\sigma=\uparrow, \downarrow)$ are real. Besides, from the analytical expressions for the above mentioned Green functions we can conclude that $V_{\sigma}^{A}=V_{-\sigma}^{B}\left(X_{\sigma}^{A}=X_{-\sigma}^{B}\right)$. Thus, we assume further that $V_{\sigma}^{A}=V_{\sigma}\left(X_{\sigma}^{A}=X_{\sigma}\right), V_{\sigma}^{B}=V_{-\sigma}\left(X_{\sigma}^{B}=X_{-\sigma}\right)$. Next, we follow exactly the same calculation scheme to find the final set of equations as in Ref. [10]. Similarly, we have used the band energies for conduction electrons by $\epsilon_{\dot{k}}$ (hopping integral $t_{i j}^{d}$ ) and for $4 f(5 f)$ electrons by $\delta \epsilon_{k}$ (hopping integral $t_{i j}^{f}$ ), where $\delta$ is the ratio of the band widths for $4 f(5 f)$ electrons and conduction band electrons. For the $B$-sublattice the corresponding Green functions can be obtained by changing $\sigma \leftrightarrow-\sigma$. We assume also (similar to Ref. [10]) the model density of states for conduction electrons in the form

$$
\rho_{0}(E)= \begin{cases}1 / W & \text { for }-W / 2 \leq E \leq W / 2 \\ 0 & \text { otherwise }\end{cases}
$$


where $W$ is the band width of the conduction band. Thus obtained implicit equations together with the constraint given by Eq. (14) form a complete set of equations to determine the chemical potential $\mu$, the average occupation numbers $\left\langle n_{A \sigma}^{f}\right\rangle,\left\langle n_{A \sigma}^{d}\right\rangle$ and the correlation functions $\left\langle d_{A \sigma}^{+} f_{A \sigma}\right\rangle$.

The numerical solution of the system of complicated seven implicit nonlinear equations not only allows to discuss the magnetic properties of the model (the possibility of appearance of the antiferromagnetic or paramagnetic phases) but also to show the temperature dependence of the average electron occupation numbers and of the chemical potential $\mu$ in the vicinity of transition (Nél) temperature. Here, since both effects (on $\left\langle n_{\sigma}^{f(d)}\right\rangle$ and on $\mu$ ) were first introduced and described in papers [11-14], we will concentrate ourselves only on the temperature dependence of the chemical potential $\mu$ (the effect of critical electron redistribution is much more difficult to measure in experiment for it needs the knowledge of the density of states and of the chemical potential $\mu$ at every temperature in the considered range). However, we will not put the special emphasis on the chemical potential alone but, to enhance the effect of chemical potential critical behaviour at critical temperatures, we introduce the chemical potential derivative (instead of recently used (Ref. [12]) relative difference of the chemical potential $\left(\mu(T)-\mu\left(T_{0}\right)\right)$ with respect to the reference value of $\mu$ at $T_{0}$ ) for it works perfectly well to distinguish critical temperatures of the considered system.

Solving numerically the system of implicit equations together with the constraint which determines the chemical potential $\mu, \mathrm{Eq}$. (14), we can calculate the order parameters (magnetizations) and the correlation functions as functions of temperature for a given parameter set. From these calculations we can read off the critical temperatures of the system and plot them as functions of $E_{f}$ level position to obtain the corresponding phase diagram. From many possibilities, in order to demonstrate the effect of critical behaviour of the chemical potential $\mu$ we have selected only two phase diagrams which we present in Fig. 1. Both phase diagrams consist of two separated areas corresponding to two different phases (namely antiferromagnetic and paramagnetic). The effect of chemical potential critical behaviour (or critical electron redistribution - see Fig. $2 \mathrm{~b}$ and the inset in Fig. 3b) can easily be demonstrated when considering the differences

$$
\Delta \mu(T)=\mu(T)-\mu\left(T_{0}\right), \quad \Delta n^{f(d)}(T)=n^{f(d)}(T)-n^{f(d)}\left(T_{0}\right),
$$

where the reference temperature $T_{0}$ is chosen to be greater than critical temperatures of the system in the considered temperature interval or by the chemical potential derivative

$$
\frac{\mathrm{d} \mu}{\mathrm{d} T} \text {. }
$$

In the present paper we put a special emphasis on the last mentioned derivative method (as it may appear to be the most promising in the experimental practice). From Fig. 2b we see that the quantities in Eq. (16) exhibit distinct kinks exactly at the same temperature as can be read off from the temperature dependence of the order parameters, Fig. 2a (the cross-section goes along $E_{f}=-2 \mathrm{eV}$ line). However, the kink in the plot of the chemical potential $\mu$ is weak and probably cannot be separated from other effects (or energetic background) in experi- 


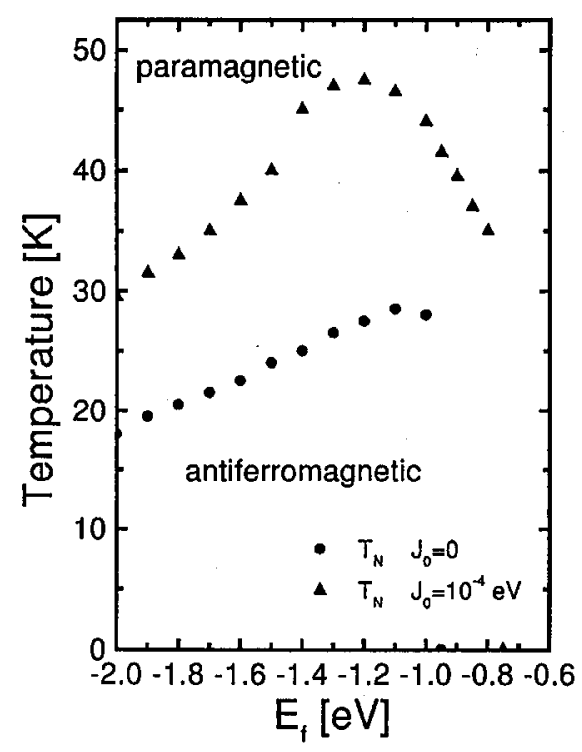

Fig. 1. The phase diagram of the system $\left(T_{\mathrm{N}}\right.$ vs. $\left.E_{f}\right)$ for $n=0.95\left(J_{0}=10^{-4} \mathrm{eV}\right.$, $\delta=10^{-5}$ - upper curve) and $n=1\left(J_{0}=0, \delta=0\right.$ - lower curve). The remaining model parameters are: $W=2 \mathrm{eV}, g=0.1 \mathrm{eV}, U_{f}=10 \mathrm{eV}, V=0.1 \mathrm{eV}$.
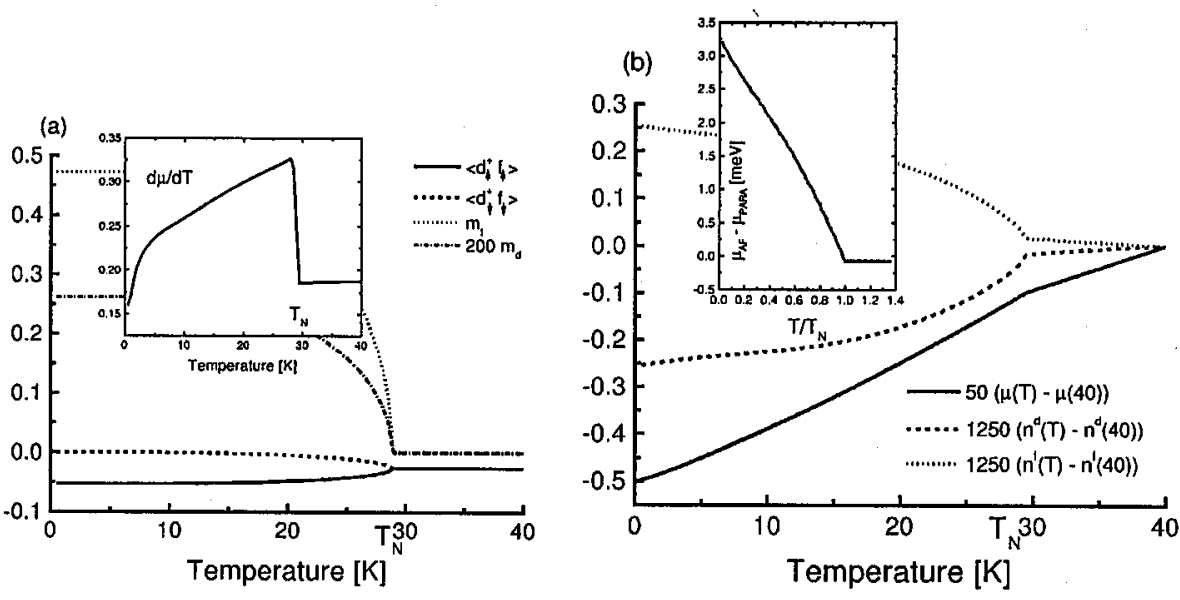

Fig. 2. (a) Magnetizations $m_{f(d)}$ and correlation functions $\left\langle d_{\alpha}^{+} f_{\alpha}\right\rangle(\alpha=\uparrow, \downarrow)$ vs. temperature; (b) the corresponding differences $\Delta \mu[\mathrm{eV}]$ and $\Delta n^{f(d)}$ vs. temperature; the inset in (a) - chemical potential derivative $\mathrm{d} \mu / \mathrm{d} T$ and the inset in (b) - the relative chemical potential $\mu_{\text {AF }}-\mu_{\text {PARA }}$ scaled by the factor $(-1)$ vs. reduced temperature $T / T_{\mathrm{N}}$. The cross-sections correspond to $E_{f}=-2 \mathrm{eV}$ line in Fig. 1 (upper curve).

ment. This drawback can be removed when we differentiate the chemical potential $\mu$ with respect to temperature $T$. The result is shown in the inset of Fig. 2a: a nicely seen jump of the chemical potential derivative points precisely to the critical temperature acquired from the condition of vanishing of the order parameters. 
(a)

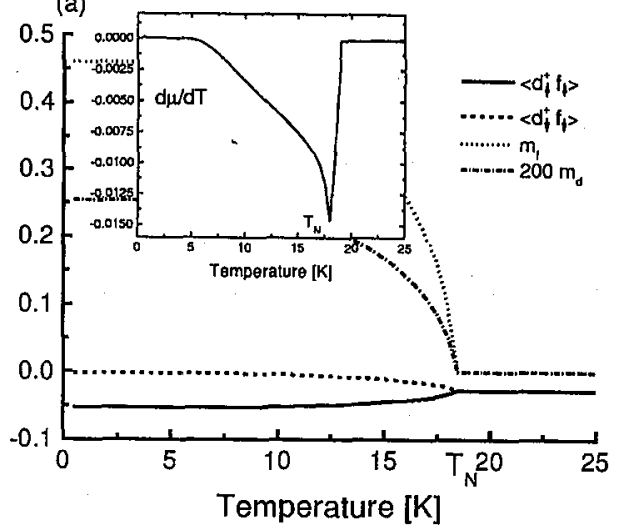

(b)

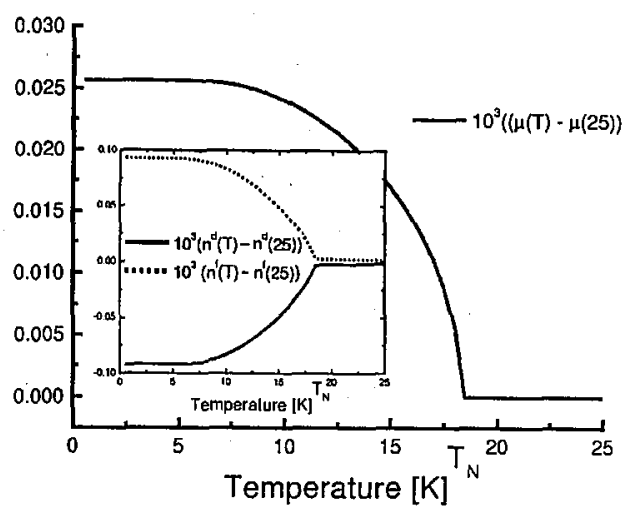

Fig. 3. (a) Magnetizations $m_{f(d)}$ and correlation functions $\left\langle d_{\alpha}^{+} f_{\alpha}\right\rangle(\alpha=\uparrow, \downarrow)$ vs. temperature; (b) the corresponding $\Delta \mu[\mathrm{eV}]$ plot; the inset in (a) - the chemical potential derivative $\mathrm{d} \mu / \mathrm{d} T$; the inset in (b) - the relative occupation numbers $\Delta n^{f(d)}$ vs. temperature. The cross-sections go along $E_{f}=-2 \mathrm{eV}$ line in Fig. 1 (lower curve).

Exactly the same phenomenon takes place in the case of the phase diagram depicted in Fig. 1 (lower curve). Also in this case distinct, well localized kinks (Fig. $3 \mathrm{~b}$ and the inset in Fig. $3 \mathrm{~b}$ ) appear precisely at the same critical temperature where the order parameters vanish (Fig. 3a). The most striking is, however, the behaviour of the chemical potential derivative which alone sharply locates, as in the first example, the critical (Néel) temperature of the considered system.

Moreover, as a supplementary method we present the calculated result which was obtained via subtraction of the chemical potential for paramagnetic reference material from the considered antiferromagnetic sample $\mu_{\mathrm{AF}}-\mu_{\mathrm{PARA}}$ (see inset in Fig. 2b). Obviously, also this "reference" method provides us with the beautiful result where the discontinuity in the slope of $\mu_{\mathrm{AF}}-\mu_{\mathrm{PARA}}$ points to the existing critical temperature (here in the reduced temperature scale: $T / T_{\mathrm{N}}$ ).

\section{Conclusions}

According to the results from the present paper, based on the model calculations in the framework of the extended $s-f$ model, and to our recent outcomes (Refs. [11-14]), we claim that the same drastic effect in chemical potential derivative would apply to the Fermi systems with temperature, concentration or (internal or external) pressure driven phase transitions. The apparently smooth and featureless curve of the temperature dependence of the chemical potential shows small but distinct kinks at phase transitions. These kinks, however, may be sometime difficult to measure (in e.g. photoemission experiments, Ref. [15]) since some other (surface) effects can take place. The experimental resolution can also impose some limitations to the application of our methods. We believe, however, that these difficulties may be omitted and the effect of critical behaviour of the chemical potential $\mu$ at points of phase transitions can be much more pronounced when we differentiate the chemical potential with respect to the actual driving force (temperature, concentration, pressure, density, etc.). 
In other words, in accordance with our present knowledge, the chemical potential derivative can serve as a new and extremely sensitive tool to detect and predict all possible phase transitions (regardless the type of transition and irrespective of the underlying mechanism), including transitions between stable and metastable phases and "exotic" states in condensed matter experimental physics. One may proceed even further and find possible applications in all quantum systems of indistinguishable Fermi or Bose particles where the notion of the chemical potential (derivative) matters.

It is worthwhile to mention that the temperature dependence of the chemical potential for high- $T_{\mathrm{c}}$ superconductor has been calculated in Ref. [16] and experimentally measured for superconducting $\mathrm{YBa}_{2} \mathrm{Cu}_{3} \mathrm{O}_{7-\delta}$ material (see Figs. 1 and 2, Ref. [17]). Almost negligibly small $(0.1 \mathrm{meV})$ kinks in the chemical potential plot at the superconducting transition temperature were of the same order of magnitude as calculated in the present paper in the case of mixed valent antiferromagnet (see inset in Fig. 2b). This observation entirely supports our suggestion that small deviations in the chemical potential are, in fact, measurable with sufficient accuracy and, accordingly, especially with the use of "first derivative" or "reference sample" methods can successfully be adopted in the hunt for phase transitions in real solids.

\section{References}

[1] J.M. Lawrance, P.S. Riseborough, R.D. Parks, Rep. Prog. Phys. 44, 1 (1981).

[2] L.M. Falicov, W. Hanke, M.B. Maple, Valence Fluctuations in Solids, North-Holland, Amsterdam 1982.

[3] Valence Instabilities, Eds. P. Wachter, H. Boppart, North-Holland, Amsterdam 1982.

[4] D. Wohlleben, B. Wittershagen, Adv. Phys. 34, 403 (1985).

[5] E. Bauer, Adv. Phys. 40, 40 (1991).

[6] D.T. Adroja, S.K. Malik, J. Magn. Magn. Mater. 100, 126 (1991).

[7] M. Matlak, W. Nolting, Z. Phys. B 55, 103 (1984).

[8] M. Matlak, W. Nolting, J. Magn. Magn. Mater. 60, 285 (1986).

[9] M. Matlak, Solid State Commun. 65, 1575 (1988).

[10] M. Matlak, M. Pietruszka, J. Magn. Magn. Mater. 153, 115 (1996).

[11] M. Matlak, M. Pietruszka, Physica C 311, 151 (1999).

[12] M. Matlak, M. Pietruszka, J. Alloys Comp. 291, 21 (1999).

[13] M. Matlak, M. Pietruszka, E. Gosławska, B. Grabiec, Kh. Eid, Phase Transit., in press.

[14] M. Matlak, M. Pietruszka, Physica B, in press.

[15] S. Hüfner, Photoemission Spectroscopy, Springer-Verlag, Berlin 1995.

[16] S. Robaszkiewicz, R. Micnas, K.A. Chao, Phys. Rev. B 20, 3915 (1982).

[17] G. Rietveld, N.Y. Chen, D. van der Marel, Phys. Rev. Lett. 69, 2578 (1992). 